\title{
Prognostic Value of Cross-sectional Anthropometric Indices on Short-term Risk of Mortality in Human Immunodeficiency Virus-infected Adults in Abidjan, Côte d'Ivoire
}

K. Castetbon, ${ }^{1-3}$ X. Anglaret, ${ }^{3,4}$ S. Touré ${ }^{3}$ G. Chêne, ${ }^{1}$ T. Ouassa,,$^{3,4}$ A. Attia, ${ }^{3,5}$ T. N'Dri-Yoman, ${ }^{3,5}$ D. Malvy, ${ }^{1,6}$ R. Salamon, ${ }^{1,3}$ and F. Dabis ${ }^{1,3}$ for the COTRIMO-CI Study Group

In sub-Saharan Africa where weight loss is very difficult to estimate, cross-sectional anthropometric indicators could be useful to predict human immunodeficiency virus (HIV)-associated mortality. The study objective was to look for threshold values of baseline body mass index, arm muscle circumference, and fat mass to predict the risk of death in HIV-infected adults included in a 1996-1998 trial of early cotrimoxazole chemoprophylaxis in Abidjan, Côte d'Ivoire (COTRIMO-Cl-ANRS 059 trial). The authors graphically determined if consecutive anthropometric categories with the closest hazards ratios of the risk of death could be clustered to obtain a unique threshold that distinctly separated two categories. When the threshold values were determined, the authors estimated the hazards ratio of mortality of this two-category model. A significant increase of mortality was observed for a body mass index of $\leq 20.3$ in men (hazards ratio $=2.6$; 95\% confidence interval $(\mathrm{Cl}): 1.4,5.0)$ and of $\leq 18.5$ in women (hazards ratio $=2.2 ; 95 \% \mathrm{Cl}: 1.05,4.5$ ) and for a fat mass of $\leq 6 \%$ in men (hazards ratio $=4.6 ; 95 \% \mathrm{Cl}: 2.3,9.4$ ) and of $\leq 18 \%$ in women (hazards ratio $=2.4 ; 95 \% \mathrm{Cl}: 1.2,4.9$ ). No simple threshold could be identified for arm muscle circumference. In Côte d'Ivoire where chemoprophylaxis of opportunistic infections has recently been recommended to be widely initiated on clinical criteria, such thresholds may help to screen patients with higher risks of mortality. Am J Epidemiol 2001;154:75-84.

body mass index; HIV infections; survival; weight loss

Weight loss is part of the definition of the human immunodeficiency virus (HIV) wasting syndrome (1) and is the unique nutritional criterion routinely used in international classification systems for HIV infection (2-4). Several studies have shown that weight loss strongly predicts morbidity and mortality in $\mathrm{HIV}$-infected $\left(\mathrm{HIV}^{+}\right)$adults (5-8). All these studies were performed on patients from industrialized countries with quite advanced immunodepression (mean $\mathrm{CD}^{+}$lymphocyte count from 86 to $281 / \mathrm{mm}^{3}$ ) (5). Most were performed on homosexual men $(5,7,8)$ and only one on women (6).

Received for publication May 10, 2000, and accepted for publication November 29, 2000.

Abbreviations: $\mathrm{Cl}$, confidence interval; COTRIMO-Cl, cotrimoxazole chemoprophylaxis in Abidjan, Côte d'Ivoire; HIV, human immunodeficiency virus; $\mathrm{HIV}^{+}$, HIV infected.

${ }^{1}$ INSERM U.330, Université Victor Segalen Bordeaux 2, Bordeaux, France.

${ }^{2}$ Centre IRD de Petit-Bassam, Abidjan, Côte d'Ivoire.

${ }^{3}$ Programme PAC-Cl, Abidjan, Côte d'Ivoire.

${ }^{4}$ Centre de Diagnostic et de Recherche sur le SIDA et les Infections Opportunistes, CHU de Treichville, Abidjan, Côte d'Ivoire.

${ }^{5}$ Service de Gastro-Entérologie, CHU de Yopougon, Abidjan, Côte d'Ivoire.

${ }^{6}$ Centre René Labusquière, Université Victor Segalen Bordeaux 2, Bordeaux, France.

Reprint requests to Dr. Katia Castetbon, INSERM U.330, Université Victor Segalen Bordeaux 2, 146 rue Léo Saignat, 33076 Bordeaux Cedex, France (e-mail: Katia.Castetbon@isped.u-bordeaux2.fr).
In sub-Saharan Africa, weight loss is often difficult to estimate for $\mathrm{HIV}^{+}$patients. When attending health care facilities for the first time, most patients either are unaware of their usual body weight or report unverifiable usual body weight. Because the CD $4^{+}$lymphocyte count and HIV viral load are also rarely available, it should be of particular interest to identify other anthropometric indicators than weight loss that could help to evaluate the risk of mortality and that could be easily measured.

Body mass index is the unique anthropometric indicator other than weight loss that has already been related to mortality in $\mathrm{HIV}^{+}$homosexual men in the Netherlands (9) and in $\mathrm{HIV}^{+}$women in Rwanda (10). However, to our knowledge, this association was never confirmed in other African women and never assessed among African men. Furthermore, body mass index, like weight loss, reflects the global nutritional status (11) but not the early and specific consequences of HIV infection on body components, namely, a reduction of fat mass $(12,13)$, accompanied in some patients by a depletion of fat-free mass $(14,15)$. Fat mass and fat-free mass could thus be hypothesized to better and earlier predict mortality than weight loss or body mass index. Several previous studies using complex and expensive techniques (bioelectric impedance analysis, total body-potassium counting) to measure body components in a limited series of patients have supported this hypothesis $(14,16,17)$. However, this remains to be confirmed in larger studies. Should these studies be performed in Africa, simpler methods that could be routinely 
implemented by clinicians (such as skinfold thicknesses and arm muscle circumference) should be used to measure body components.

We report an analysis of the prognostic value of body mass index, arm muscle circumference, and skinfold thicknesses on mortality in $\mathrm{HIV}^{+}$men and women included in a randomized placebo-controlled trial of prevention of opportunistic infections by trimethoprim-sulfamethoxazole in Abidjan, Côte d'Ivoire. This trial has demonstrated the efficacy of such a prophylaxis initiated at an early stage of HIV disease in African patients (18).

\section{MATERIALS AND METHODS}

\section{Patients}

The present study was performed on patients included in a double-blind placebo-controlled trial conducted in Abidjan, Côte d'Ivoire, from April 1996 to March 1998. This study assessed the efficacy of cotrimoxazole prophylaxis in reducing severe clinical events in HIV-infected adults recruited at early stages of HIV-1 infection (COTRIMO-CI-ANRS 059 trial). The study population and medication have been described elsewhere (18). In brief, patients whose HIV infection was diagnosed in routine consultations at five urban community health centers of Abidjan were included if they met the following criteria: aged 18 years or more, HIV-1 or HIV-1 plus HIV-2 dual infection, clinical stage 2 or 3 of the World Health Organization staging system for HIV infection and disease (2), and written consent. Reasons for exclusion were pregnancy, breastfeeding, prior history of intolerance to any sulfonamide, hemoglobin level less than $7 \mathrm{mg} / \mathrm{dl}$, platelet count less than $75,000 / \mathrm{mm}^{3}$, absolute neutrophil count less than $750 / \mathrm{mm}^{3}$, and renal or hepatic failure. The lymphocyte $\mathrm{CD}^{+}$count was not used as an inclusion/exclusion criterion but was documented by flow cytometry after randomization in all included patients. Subjects were randomly assigned to receive daily either cotrimoxazole $(800 \mathrm{mg}$ of sulfamethoxazole and $160 \mathrm{mg}$ of trimethoprim) in a single tablet or a matching placebo. No patient had a history of chemoprophylaxis against tuberculosis or any other opportunistic infections at enrollment in the trial. Before the trial and during trial follow-up, no patients received antiretroviral treatment.

\section{Measurements}

Baseline demographic, clinical, and biologic data were recorded through standardized questionnaires, including a physical examination and measurement of the $\mathrm{CD}^{+}$count. Three physicians, trained by one nutritionist, performed anthropometric measurements in a standardized fashion: weight (measured to the nearest $0.2 \mathrm{~kg}$, on an electronic scale, patient dressed but without shoes); height (measured to the nearest $1 \mathrm{~cm}$, on a wall scale, patient without shoes and with joined feet); mid-upper arm circumference (measured to the nearest $5 \mathrm{~mm}$, on the half left arm); and skinfold thickness at four sites (bicipital, tricipital, subscapular, and suprailiac) measured to the nearest $0.2 \mathrm{~mm}$ with a caliper
(SiberHegner \& Cie. S.A., Miribel, France). We then computed the body mass index as the ratio of weight $(\mathrm{kg})$ to height $(\mathrm{m})$ squared, the arm muscle circumference as the mid-upper arm circumference $-\pi \times$ the tricipital skinfold (mm) (19), and the percentage of fat mass according to the Durnin-Womersley equation using values of the above four skinfold thicknesses, age, and gender (20). During the initial interview, patients were systematically asked for their usual body weight defined as their weight before they became symptomatic for HIV disease. For patients who selfreported a usual body weight, the percentage of weight loss was defined as $100 \times$ (usual body weight - inclusion body weight)/usual body weight.

\section{Follow-up}

After randomization, patients were asked to attend monthly scheduled visits. Within the interval, they were asked to visit the medical team at the trial center as soon as they had medical problems. All medications, explorations, hospitalizations, and transportation were offered on a free basis. All clinical events (including death) were referred for independent review by an event documentation committee blinded to the treatment group. Vital status was systematically investigated for patients who did not keep scheduled appointments through home visits and telephone calls and by screening the obituary sections of the national newspapers.

\section{Statistical analysis}

In this report, we analyzed patients with complete anthropometric data at entry into the study and available follow-up data. The follow-up duration was defined as the interval between the date of inclusion and the date of death, or the date of the last contact with the trial team if before the end of the trial (March 17, 1998), or the date of the end of the trial if a contact with the trial team was recorded after March 17, 1998. Comparisons of means were performed using the Student $t$ test or the Student $t$ test for unequal variances if Bartlett's test for the equality of variances was $<0.05$. For comparisons of proportions, the chi-squared test was used. Subsequent analyses were performed with men and women separately because of the inherent difference of the distribution in body components (19).

The following nonanthropometric baseline characteristics were studied in relation to the risk of death through univariate analyses by gender using proportional hazards models: age; any paid activity; school level (patients never schooled or at primary school level vs. patients with at least secondary school level); $\mathrm{CD}^{+}$count $\left(\mathrm{CD} 4^{+}\right.$of $<200 / \mathrm{mm}^{3}$, $200-500 / \mathrm{mm}^{3}$, and $>500 / \mathrm{mm}^{3}$ ); World Health Organization clinical stages; and treatment groups (cotrimoxazole vs. placebo). Subsequent multivariate analyses included all baseline nonanthropometric variables related to mortality in the above univariate analyses with a $p<0.25$.

To determine the thresholds of substantial increase in the risk of death, we used the analysis strategy presented in figure 1 for each anthropometric indicator and each gender. Our strategy combined an approach derived from cluster 


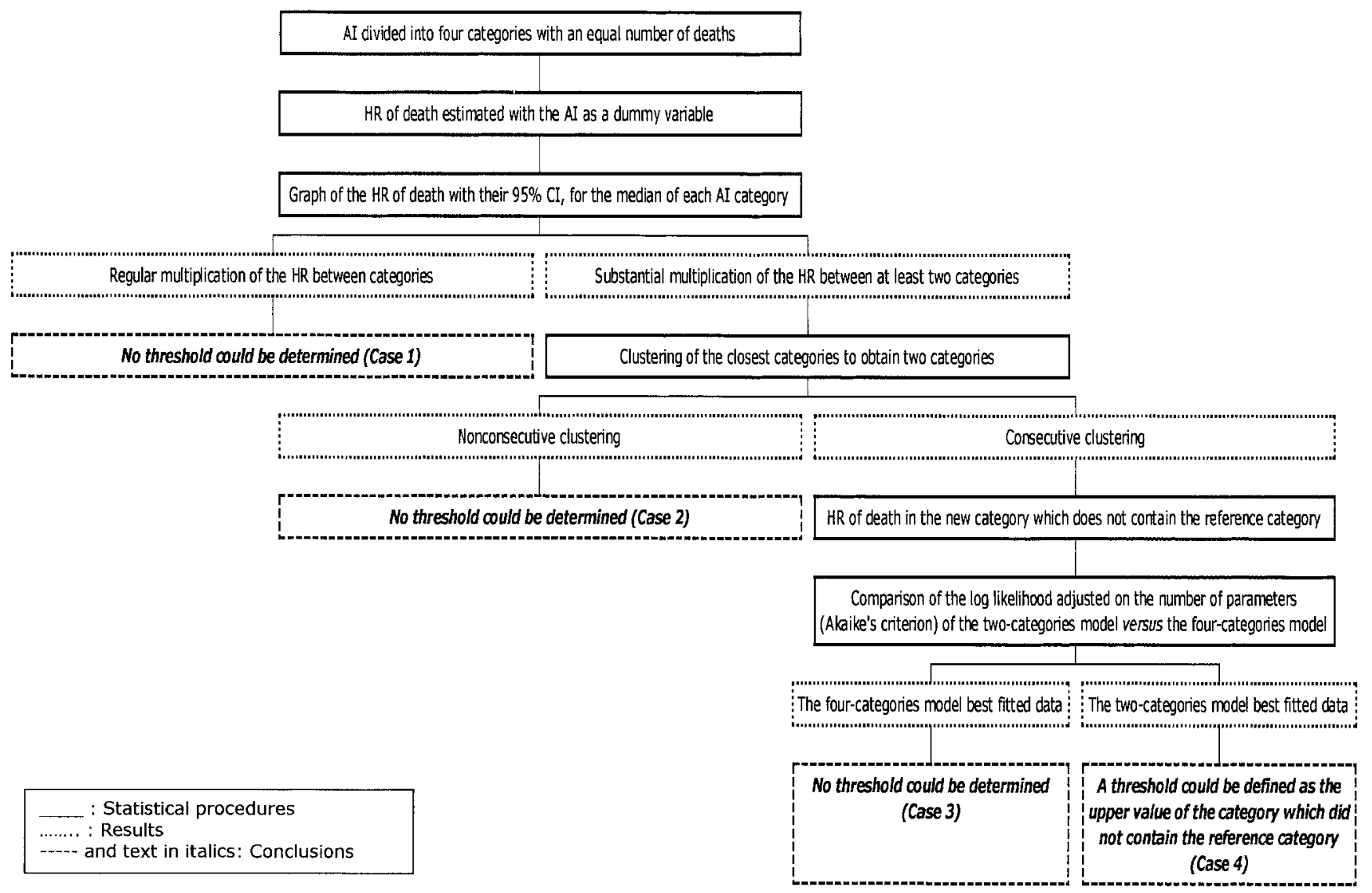

FIGURE 1. Analysis strategy for determination of anthropometric thresholds to distinguish survival in human immunodeficiency virus-infected adults in Abidjan, Côte d'Ivoire, 1996-1998. Al, anthropometric indicator; HR, hazards ratio estimated by the proportional hazards model in reference to the anthropometric category with the highest values and adjusted on covariates; $\mathrm{Cl}$, confidence interval.

analysis (21) and from the principles of modeling in a prognosis context (22). After dividing the anthropometric indicator into four categories, each of which contained an equal number of deceased patients, we estimated the hazards ratio for each dummy variable thus created using as the reference class the category with the highest value of the indicator. Graphical representation of the hazards ratio was used to estimate whether the increase in the risk of death was constant or if a substantial increase of this risk was appreciable between two categories. When the latter was the case, the closest categories were combined to obtain two categories, the anthropometric threshold of mortality being defined by the highest value of the category not containing the reference values. The Akaike Information Criterion was used to compare the fit of the data for the models with two and four categories. When no threshold could be determined because of a log-linear relation (case 1 in figure 1) or nonconsecutive clustering (case 2 in figure 1), the results of the model that included the anthropometric indicator as a continuous variable were presented. When no threshold could be defined because the four-category model best fit the data (case 3 in figure 1), the results of this latter model were presented. When a threshold could be determined (case 4 in figure 1), we presented the survival using Kaplan-Meier life tables according to the baseline nutritional status around this threshold.

\section{RESULTS}

\section{Baseline characteristics}

From April 23, 1996, to March 17, 1998, 545 patients were assigned at random to the COTRIMO-CI-ANRS 059 trial (18), of whom 35 (6 percent) were excluded from the analyses for the present study: four because they were subsequently confirmed to be HIV-2 $2^{+}$only, 16 because they had incomplete anthropometric data at inclusion, and 15 because they had no available follow-up information. Follow-up data and data on the documentation of vital status of patients enrolled in the ANRS 059 trial have been previously reported elsewhere (18). Among the 510 patients analyzed in the present study, 86 (17 percent) were known to be deceased at trial termination, 389 (76 percent) had been seen at least once by the trial team within the last month, and for the remaining 35 (7 percent) the time from the last contact with the trial team to the trial termination was more than 1 month. 
Among the 510 patients analyzed, 58 percent were women, 50 percent had a paid activity, and 56 percent were never schooled or had reached a primary school level. The mean age was 32.8 (standard deviation, 8.9) years. At entry into the trial, 34 percent of the patients were classified at World Health Organization clinical stage 2, 60 percent at stage 3 , and 6 percent at stage 4 . The mean $\mathrm{CD}^{+}$lymphocyte count was 325 (standard deviation, 263)/ $/ \mathrm{mm}^{3}$, while 39 percent of the patients had a $\mathrm{CD}^{+}$lymphocyte count of $<200 / \mathrm{mm}^{3}, 41$ percent had a count of $200-500 / \mathrm{mm}^{3}$, and 20 percent had a count of $>500 / \mathrm{mm}^{3}$.

The following baseline characteristics differed significantly between men and women: age (36.2 years vs. 30.4 years, $p<10^{-4}$ ), percentage of patients with paid activity (64 percent vs. 40 percent, $p<10^{-4}$ ), percentage who were never schooled or at a primary school level (41 percent vs. 68 percent, $\left.p<10^{-4}\right)$, and mean $\mathrm{CD} 4^{+}$count $\left(275 / \mathrm{mm}^{3}\right.$ vs. $360 / \mathrm{mm}^{3}, p=0.0003$ ). The proportion of patients at World Health Organization stages 2, 3, and 4 did not differ significantly between men and women (stage 2: 30 percent vs. 37 percent; stage 3: 62 percent vs. 58 percent; $p=0.07$ ). As expected, distributions of demographic, biologic, and clinical data were comparable at baseline between randomization groups: cotrimoxazole or placebo (data not shown) (18).

Baseline anthropometric characteristics are described in table 1. Weight loss could be estimated in 75 percent of patients (men: 91 percent, women: 64 percent; $p<10^{-4}$ ). Cross-sectional nutritional indicators recorded at baseline were comparable in patients who did not know their usual body weight and in those who did, with the exception of mean weight (50.7 kg vs. $53.3 \mathrm{~kg} ; p=0.02$ ) and mean body mass index (19.9 vs. $20.7 ; p=0.01)$ in women, suggesting that women who did not know their usual body weight presented a poorer nutritional status than women who knew it.

Although 90 percent of patients with known usual body weight were considered to have weight loss, moderate or severe malnutrition was infrequent in this group of patients: only 2 percent had severe thinness (body mass index of $<16), 79$ percent had a normal corpulence (body mass index of $\geq 18.5$ ), and 70 percent had a normal mid-upper arm circumference of $>243 \mathrm{~mm}$ for men and of $>232 \mathrm{~mm}$ for women according to World Health Organization standards (19). The mean reported weight loss was significantly higher in men than in women. All other anthropometric variables were distributed as expected according to gender: the mean body mass index was comparable for men and women, the mean arm muscle circumference was higher in men than in women, and the mean fat mass was lower in men than in women (table 1).

\section{Prognostic value on survival of thresholds of nutritional indicators}

Covariates. The 510 patients included in the analysis experienced a mean follow-up of 9.9 months (421 personyears). During this follow-up period, 86 patients died, 51 men and 35 women. The following variables were related to survival with a statistical significance of less than 0.25 : in

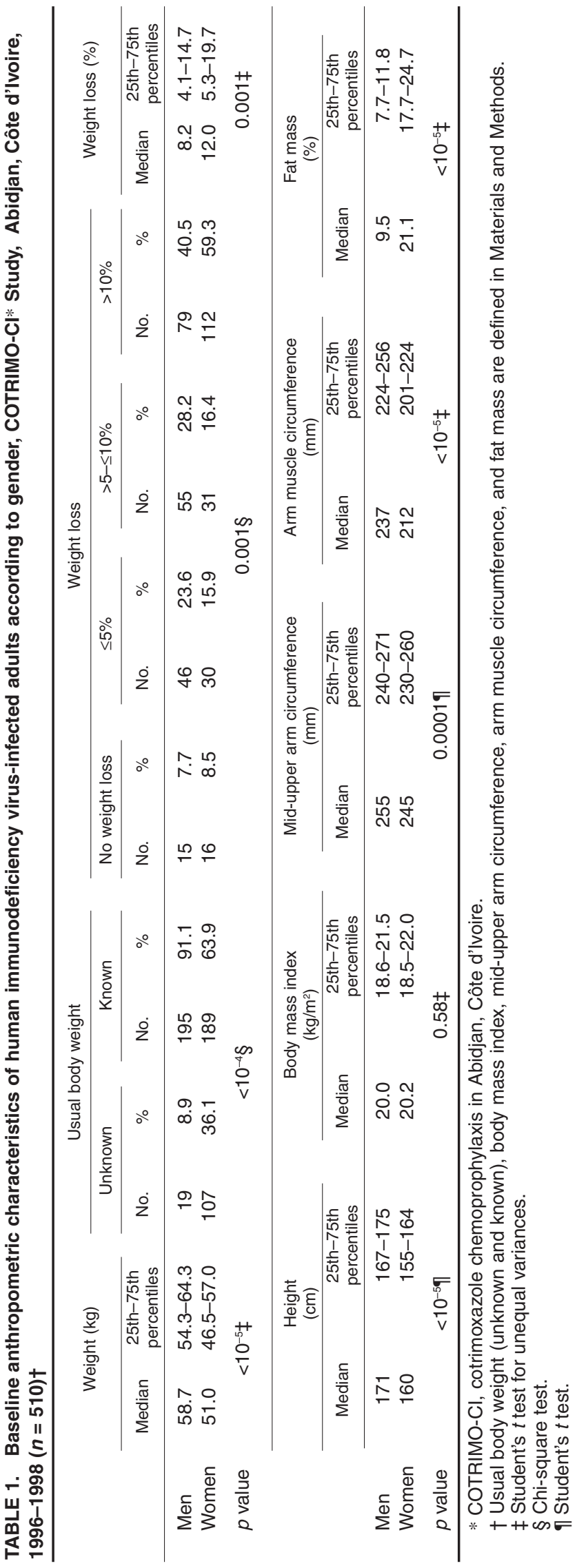

Am J Epidemiol Vol. 154, No. 1, 2001 
men, the $\mathrm{CD}^{+}$count (compared with the $\mathrm{CD} 4^{+}$count of $>500 / \mathrm{mm}^{3}$ : hazards ratio $=6.2(95$ percent CI: $0.8,47.6)$ for a CD4 ${ }^{+}$count of $200-500 / \mathrm{mm}^{3}, p=0.08$; and hazards ratio $=$ 19.2 (95 percent CI: $2.6,140.2$ ) for a $\mathrm{CD}^{+}$count of $\left.<200 / \mathrm{mm}^{3}, p=0.004\right)$; World Health Organization clinical stage (compared with stage 2 : hazards ratio $=1.9$ (95 percent CI: $0.9,3.8), p=0.08$, for stage 3 ; and hazards ratio $=$ 2.9 (95 percent CI: $1.1,7.8$ ), $p=0.03$, for stage 4 ); and age (hazards ratio $=1.2(95$ percent CI: $0.9,1.6)$ for 10 years higher, $p=0.17)$. In women, the respective values were as follows: $\mathrm{CD}^{+}$count (hazards ratio $=4.8(95$ percent $\mathrm{CI}$ : $0.6,39.2), p=0.14$, for a CD4 $4^{+}$count of $200-500 / \mathrm{mm}^{3}$, and hazards ratio $=37.2$ (95 percent CI: 5.0, 276.7), $p<10^{-3}$, for a CD4 $4^{+}$count of $<200 / \mathrm{mm}^{3}$ ) and World Health Organization clinical stage (hazards ratio $=2.6(95$ percent CI: $1.1,6.1), p=0.03$, for stage 3 , and hazards ratio $=8.7$ (95 percent CI: 2.7, 27.7), $p<10^{-3}$, for stage 4 ). The statistical significance of the relation between treatment group (cotrimoxazole vs. placebo) and mortality was 0.83 in men and 0.39 in women. The treatment group was thus not taken into account in subsequent multivariate analyses.

Weight loss. In a comparison of men with known usual body weight and no weight loss or a weight loss of $\leq 5$ percent $(n=60)$, the hazards ratios of death were 1.8 (95 percent CI: $0.7,4.5)(p=0.19)$ for men with a $>5-\leq 10$ percent weight loss $(n=55)$ and 2.9 (95 percent CI: $1.2,6.8)(p=$ $0.01)$ for men with a weight loss of $>10$ percent $(n=80)$. In women, the hazard ratios were 6.9 (95 percent CI: $0.6,83.9)$ $(p=0.13)$ for a weight loss of $>5-\leq 10$ percent $(n=30)$ and 18.6 (95 percent CI: $1.8,189.8)(p=0.01)$ for a weight loss of $>10$ percent $(n=113)$. In these mutivariate analyses, the $\mathrm{CD}^{+}$count remained significantly related to survival. For example, in men, the hazards ratio $=7.0$ (95 percent CI: 0.9, 54.4) $(p=0.06)$ for a $\mathrm{CD} 4^{+}$count of $200-500 / \mathrm{mm}^{3}$; the hazards ratio $=18.9(95$ percent CI: $2.5,142.3)(p=0.004)$ for a CD4 $4^{+}$count of $<200 / \mathrm{mm}^{3}$.

Body mass index, arm muscle circumference, and fat mass in men. Figure 2 shows the relation between the hazards ratio of death and the median value of body mass index (figure 2, top), arm muscle circumference (figure 2, middle), and fat mass (figure 2, bottom). We further grouped into a unique category the first three categories for body mass index and arm muscle circumference and for the last three categories for fat mass. This allowed us to define only two categories of patients for each nutritional indicator, distinguished by the following thresholds: 20.3 for body mass index, $250 \mathrm{~mm}$ for arm muscle circumference, and 6 percent for fat mass. Comparison of the log-likelihood, weighted for the number of parameters, of the two-category models versus the four-category models showed that the two-category models best fit the data (body mass index: -232.5 vs. -234.0; arm muscle circumference: -235.4 vs. -237.2 ; and fat mass: -230.3 vs. -230.7 , respectively). When comparing survival acording to these thresholds (table 2), we found that body mass index and fat mass were significantly related to survival (hazards ratio $=2.6$ (95 percent CI: $1.4,5.0)$ for a body mass index of $\leq 20.3$ and hazards ratio $=4.6$ ( 95 percent CI: $2.3,9.4$ ) for a fat mass of $\leq 6$ percent), while the relation did not reach significance for an arm muscle cir- cumference of $\leq 250 \mathrm{~mm}$ (hazards ratio $=2.0$ (95 percent CI: $0.95,4.1)$ ). In all of these multivariate analyses, the $\mathrm{CD}^{+}$lymphocyte count remained significantly related to survival (e.g., in the model with body mass index, the hazards ratio $=5.9(95$ percent CI: $0.77,45.5)$ for a $\mathrm{CD}^{+}$count of $200-500 / \mathrm{mm}^{3}(p=0.09)$; the hazards ratio $=17.9(95$ percent CI: $2.4,132.1)$ for a CD $4^{+}$count of $<200 / \mathrm{mm}^{3}(p=$ $0.005)$ ), unlike the other studied covariates (age and World Health Organization clinical stage). When performing multivariate analysis that included body mass index, arm muscle circumference, fat mass, and the covariates, we found that the fat mass threshold provided the best prediction of the risk of death (hazards ratio $=3.7 ; 95$ percent CI: 1.8 , 7.7), whereas the body mass index (hazards ratio $=1.9 ; 95$ percent CI: $0.90,4.20$ ) and arm muscle circumference (hazards ratio $=1.3 ; 95$ percent CI: $0.55,2.97$ ) thresholds were not associated with mortality.

Body mass index, arm muscle circumference, and fat mass in women. It was not possible to determine a threshold for arm muscle circumference (figure 3, middle), because the closest categories were the first and the third. The model that included arm muscle circumference as a continuous variable showed that it was not associated with survival (hazards ratio $=0.91 ; 95$ percent CI: $0.76,1.09$ ) ( $p=0.31$ for $10 \mathrm{~mm}$ higher). For body mass index (figure 3 , top) and fat mass (figure 3, bottom), we grouped the first two classes and the corresponding thresholds were 18.5 percent and 18 percent, respectively. For body mass index, the weighted log-likelihoods were -156.5 for the model with two nutritional categories and -158.4 for the model with four nutritional categories; for fat mass, they were -155.5 and -157.4 , respectively. Survival analyses showed that we could distinguish the survival of women when classifying them according to the baseline nutritional values around the determined thresholds above (table 2). The $\mathrm{CD}^{+}$count remained significantly associated with survival in these models (e.g., in the model with body mass index: the hazards ratio $=5.0(95$ percent CI: $0.61,45.5)$ for a $\mathrm{CD}^{+}$count of $200-500 / \mathrm{mm}^{3}(p=0.13)$; the hazards ratio $=32.0(95$ percent CI: 4.2, 242.7) for a CD $4^{+}$count of $<200 / \mathrm{mm}^{3}$ ( $p=$ $0.001)$ ), unlike the World Health Organization stage. In multivariate analysis that included the body mass index, fat mass, and covariates, the fat mass threshold was the best predictor of mortality (hazards ratio $=2.0(95$ percent $\mathrm{CI}$ : $0.99,4.36)$ ).

\section{DISCUSSION}

To our knowledge, this is the first study that assessed the prognostic value of anthropometric indicators on survival in African HIV-1-infected men and women. Our population was part of a group of HIV-1-infected adults included in a trial of early cotrimoxazole primary chemoprophylaxis (18). We analyzed available data from 94 percent of the patients enrolled in this trial and followed up during a mean of 10 months. We found that the nutritional indicators at baseline had a prognostic value on survival independently from the $\mathrm{CD} 4{ }^{+}$count measured at the same time. We determined limits for each nutritional variable that were correlated with 

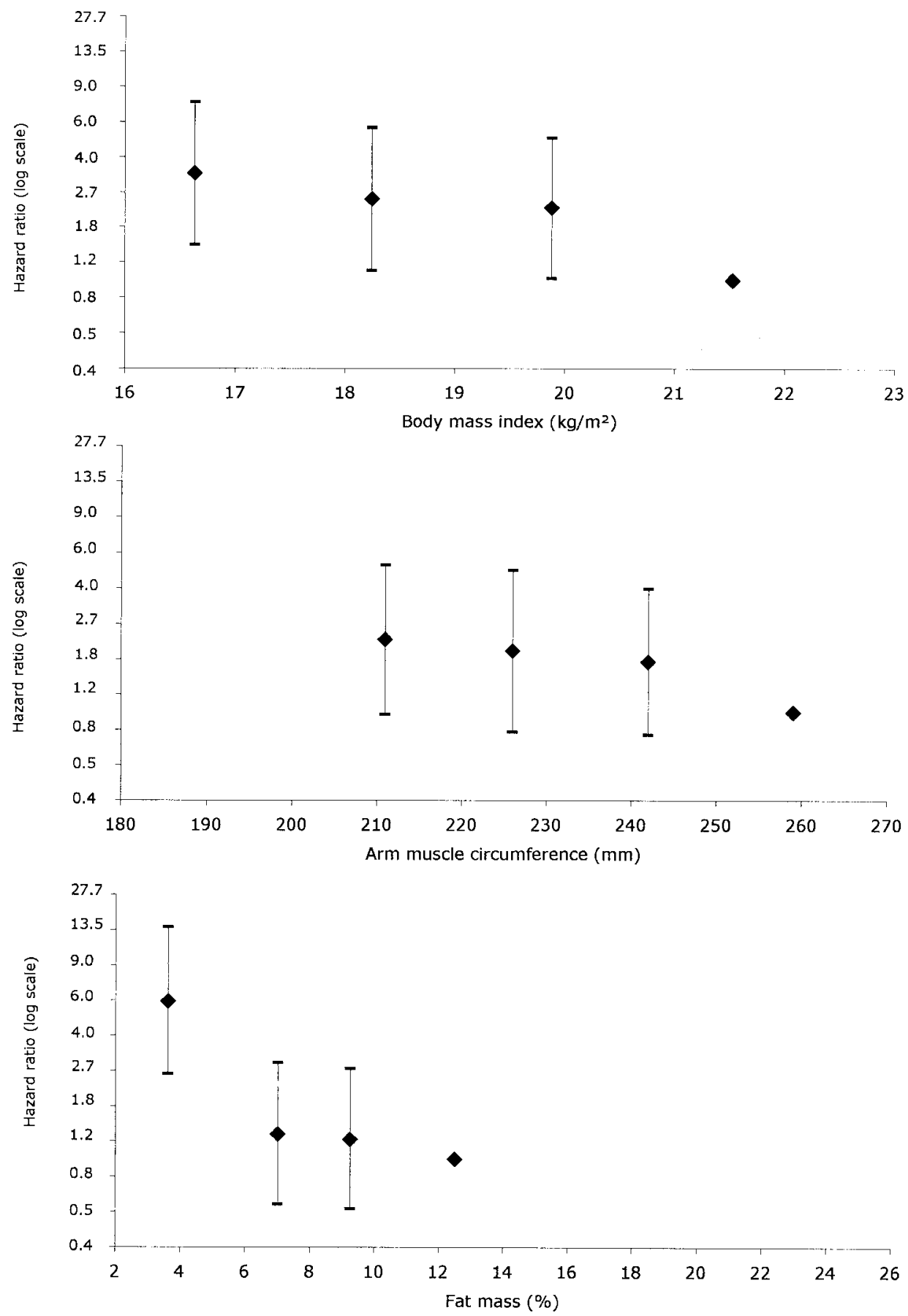

FIGURE 2. Relation between hazards ratios of death (Napierian logarithmic scale) and baseline values of body mass index (top), arm muscle circumference (middle), and fat mass (bottom) in human immunodeficiency virus-infected men in Abidjan, Côte d'Ivoire, 1996-1998 ( $n=$ 214). The hazards ratios of death were estimated from proportional hazards models that included each class of the anthropometric variables except the highest and that were adjusted for the lymphocyte CD4+ count at baseline divided into three classes, the World Health Organization clinical stage, and the age (for 10 years higher). Bars, confidence interval.

survival: 20.3 for body mass index in men and 18.5 in women and 6 percent for fat mass in men and 18 percent in women. The arm muscle circumference in men and in women did not have a prognostic value on survival in our population.
Interpretation of our findings must take into account several issues. First, the reference for each anthropometric variable was defined as the category containing the highest values. Our findings thus closely depend on our population. Included patients were screened according to inclusion cri- 
TABLE 2. Kaplan-Meier estimation of the probability of survival according to the limits of anthropometric variables and risk estimates in proportional hazards models in human immunodeficiency virus-infected adults in Abidjan, Côte d'Ivoire, 1996-1998 $(n=510)$

\begin{tabular}{|c|c|c|c|c|c|c|c|c|}
\hline & \multirow{3}{*}{ No.* } & \multicolumn{4}{|c|}{ Survival at } & \multirow{3}{*}{$\begin{array}{c}\text { Hazards } \\
\text { ratio }\end{array}$} & \multirow{3}{*}{$95 \% \mathrm{Cl}$} & \multirow{3}{*}{$\begin{array}{c}p \\
\text { value }\end{array}$} \\
\hline & & \multicolumn{2}{|c|}{6 months } & \multicolumn{2}{|c|}{12 months } & & & \\
\hline & & Probability & $95 \% \mathrm{Cl}$ & Probability & $95 \% \mathrm{Cl}$ & & & \\
\hline \multicolumn{9}{|c|}{ Body mass index $\left(\mathrm{kg} / \mathrm{m}^{2}\right)$} \\
\hline \multicolumn{9}{|c|}{ Men } \\
\hline$\leq 20.3$ & 120 & 82.8 & $74.3,88.7$ & 66.8 & $56.6,75.3$ & 2.62 & $1.37,5.00 \ddagger$ & 0.004 \\
\hline$>20.3$ & 74 & 93.1 & $85.3,96.8$ & 87.0 & $76.9,92.8$ & & & \\
\hline \multicolumn{9}{|l|}{ Women } \\
\hline$\leq 18.5$ & 72 & 92.7 & $83.3,96.9$ & 66.4 & $50.7,78.1$ & 2.17 & $1.05,4.47 \S$ & 0.04 \\
\hline$>18.5$ & 224 & 96.1 & $92.0,98.1$ & 91.2 & $85.1,94.9$ & & & \\
\hline \multicolumn{9}{|c|}{ Arm muscle circumference $(\mathrm{mm}) \uparrow$} \\
\hline \multicolumn{9}{|c|}{ Men } \\
\hline$\leq 250$ & 149 & 84.0 & $76.6,89.1$ & 70.8 & $61.7,78.1$ & 1.97 & $0.95,4.08 \ddagger$ & 0.07 \\
\hline$>250$ & 65 & 95.1 & $85.5,98.4$ & 86.4 & $73.4,93.4$ & & & \\
\hline \multicolumn{9}{|c|}{ Fat mass (\%) } \\
\hline \multicolumn{9}{|c|}{ Men } \\
\hline$\leq 6$ & 21 & 55.0 & $31.3,73.5$ & 40.7 & $18.0,62.5$ & 4.63 & $2.29,9.38 \ddagger$ & $<10^{-3}$ \\
\hline$>6$ & 193 & 90.9 & $85.5,94.3$ & 79.4 & $72.1,85.1$ & & & \\
\hline \multicolumn{9}{|l|}{ Women } \\
\hline$\leq 18$ & 81 & 91.2 & $81.5,96.0$ & 66.0 & $50.9,77.4$ & 2.43 & $1.21,4.88 \S$ & 0.01 \\
\hline$>18$ & 215 & 96.8 & $93.0,98.6$ & 91.9 & $85.9,95.4$ & & & \\
\hline
\end{tabular}

teria in a randomized clinical trial and, thus, may not represent the population of HIV-infected adults living in Abidjan. However, they were recruited in routine ambulatory consultations of urban community health centers and may, thus, be considered as more representative of the unscreened population of symptomatic HIV-infected adults looking for care than HIV-infected inpatients hospitalized with advanced HIV disease. Second, the context of a therapeutic trial, in which half of the patients received cotrimoxazole and half a placebo, is a potential limit. However, we verified that there was no interaction on mortality between the treatment groups and the nutritional status at inclusion. Third, our reference was the nutritional measurement at inclusion in a prevalent cohort, without information on the date of infection. However, we measured the $\mathrm{CD}^{+}$lymphocyte count at baseline and verified that the anthropometric indicators were associated with the risk of mortality independently from the $\mathrm{CD}^{+}{ }^{+}$count. Fourth, our analysis compares groups of patients classified at a given time and does not estimate prospectively the change in the risk of death according to the evolution of the anthropometric indicators, especially the changes below or above the defined thresholds. For this purpose, treatment of the anthropometric variables as timedependent variables would be required (9). However, as an initial step and from a pragmatic standpoint, our study based on the initial measure is justified as it corresponds to the situation of most African settings where no follow-up data are available. Further studies should now validate these anthropometric thresholds in other samples of patients.

In our population, we verified that weight loss had a prognostic value on mortality, although 9 percent of men and 36 percent of women did not know their usual body weight. This difference possibly reflects a lower access to care in women than in men. In patients with known usual body weight, the estimated mean weight loss was higher in women than in men, although their body mass index was comparable and the $\mathrm{CD}^{+}$count was higher in women, this latter being related to their less advanced stage of HIV infection than men. We supposed that women overestimated their usual body weight, possibly in reference to a weight recorded during or immediately after a previous pregnancy. These observations emphasize the need for cross-sectional anthropometric references other than weight loss, inasmuch as we observed that women who did not know their usual body weight were at a higher risk of malnutrition. Although the duration of follow-up was shorter in our study, our findings are comparable with those of three previous studies mainly performed in homosexual men from the United States. A first one (8) showed that mortality was 2.5 times higher in patients who experienced a weight loss of $>10$ percent than in patients with no weight loss $(p \leq 0.001)$. In a second one (7), the median survival times decreased significantly when weight loss increased $(p=0.0001)$. In a third one (5), patients with a weight loss of $>10$ percent had a 

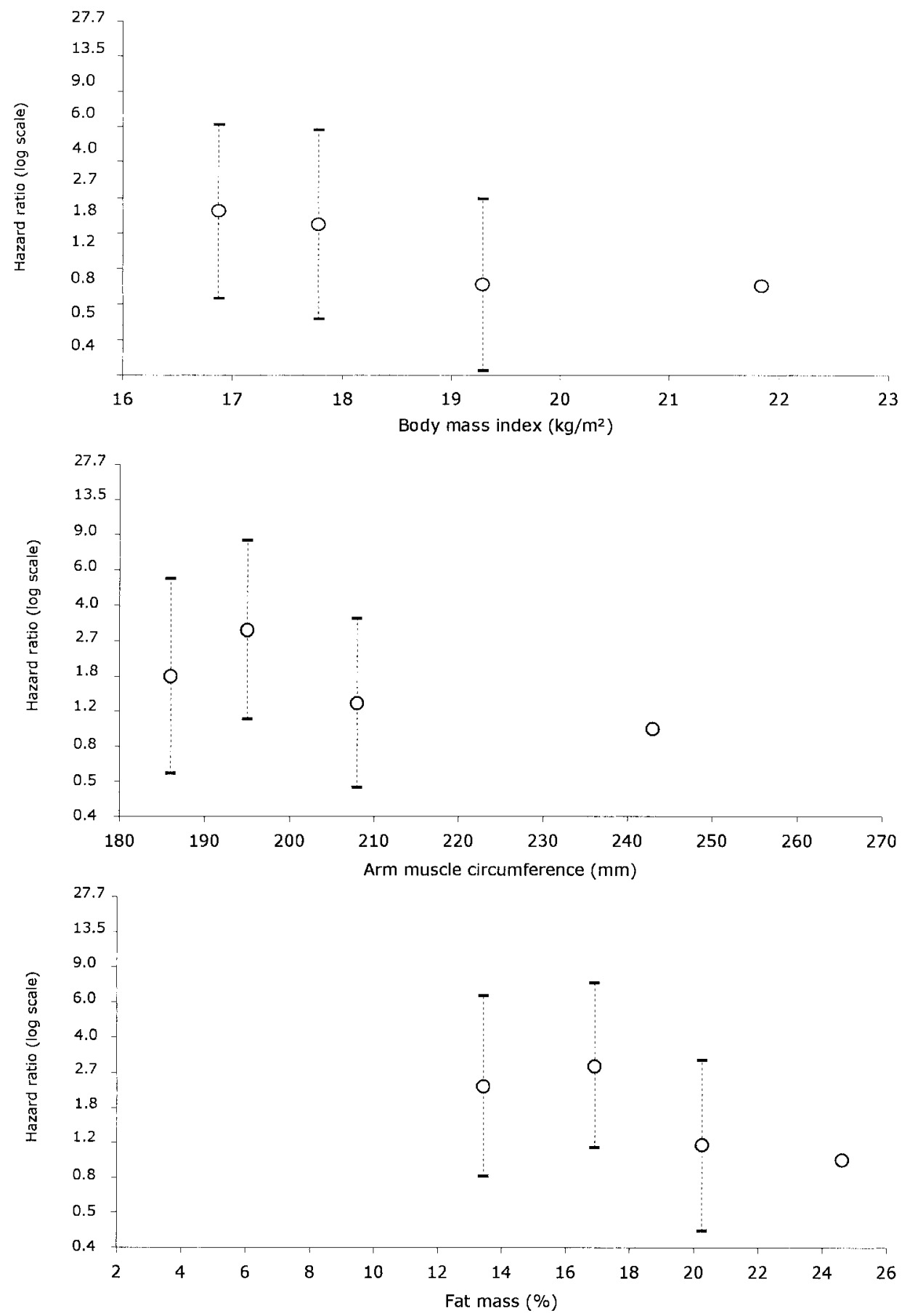

FIGURE 3. Relation between hazards ratios of death (Napierian logarithmic scale) and values of body mass index (top), arm muscle circumference (middle), and fat mass (bottom) in human immunodeficiency virus-infected women in Abidjan, Côte d'Ivoire, 1996-1998 ( $n=296)$. The hazards ratios of death were estimated from proportional hazards models that included each class of the anthropometric variables except the highest and that adjusted for the lymphocyte $\mathrm{CD4}^{+}$count at baseline divided into three classes and the World Health Organization clinical stage. Bars, confidence interval.

mortality 8.3 times higher, on average, than patients with a weight loss of $\leq 10$ percent $(p<0.002)$. All of these estimations were adjusted for $\mathrm{CD}^{+}$count, as ours. This underlines the notion that nutritional status is associated with prognosis, independently from immune status in HIV infection, and the interest of a nutritional component in a global biologic and clinical evaluation.

The main objective of our study was to evaluate the contribution of cross-sectionally measured anthropometric indicators other than weight loss to the prediction of survival. Of 
the studied nutritional indicators, only body mass index was previously assessed with this aim in a study performed in HIV-infected women from Rwanda (10). In this previous study, women with a body mass index of $\leq 21$ experienced a mortality rate 2.3 times higher, on average, than did women with a body mass index of $>21$. For a comparable hazards ratio of death, our threshold of body mass index in women was surprisingly lower (and closer to the reference value in the non-HIV population (19)), while our threshold in men was comparable with the threshold found in the Rwandese women. One could formulate the following hypothesis to explain this fact: women in our study had a better initial immunologic status than did men and, although body mass index was globally shown to predict mortality independently from the $\mathrm{CD}^{+}$count, the level of a calculated threshold probably depends on the stage of the disease in a selected population. As $\mathrm{CD}^{+}$counts were not performed in the Rwandese study, we could not compare the immunologic status of the population of the two studies. However, in our study, the differences between men and women in the initial immune stages and in the calculated body mass index thresholds related with mortality support this hypothesis. Despite this limitation, body mass index is clearly confirmed to be an easy-to-measure alternative to weight loss to predict survival.

The two other nutritional indicators assessed in this study remain of interest. Although the depletion of body cell mass is usually considered more critical for survival than the depletion of fat mass (14), the increased risk of death for impaired arm muscle circumference was lower than for reduced fat mass, and the association of the arm muscle circumference value with survival was not significant. Moreover, when performing analyses with body mass index, arm muscle circumference, and fat mass together, we found that fat mass was the most strongly related to survival. These findings could be explained by the relatively early stage of HIV disease in our population, in whom the depletion of fat mass could have been more important than the depletion of fat-free mass, as previously described (12). Moreover, the anthropometric estimation of the fat-free mass imperfectly reflects body cell mass, which was evaluated through accurate total ${ }^{40} \mathrm{~K}$ in the study showing the relation between survival and nutritional status (14). Statistical analyses performed with the bone-free arm muscle area (23) instead of the arm muscle circumference showed the same findings as in our sample. Finally, a low percentage of fat mass could be as prejudicial to survival in HIV-infected adults as a low body cell mass level, and fat mass could thus be more interesting to monitor at early stages of HIV disease than arm muscle circumference. In settings where calipers are not easily accessible, body mass index evaluation remains useful.

We observed that distributions of arm muscle circumference and fat mass values differed greatly between men and women, and we found very different thresholds predicting survival in men and women for fat mass (6 percent vs. 18 percent). These findings are compatible with the present knowledge about gender differences in general and HIV-associated nutritional alterations $(24,25)$. However, the observed differ- ence in the fat mass threshold between genders could also have been partly related to the highest level of immunodepression of men in our population as stated above for body mass index. The estimated fat mass threshold in men was particularly low (6 percent) in the limit acceptable with survival. With one quarter of the men in our study presenting fat mass less than 7.7 percent (table 1), these levels do not seem exceptional. Even if our results were adjusted for $\mathrm{CD}^{+}$count, it remains to elucidate whether different thresholds should be used at different states of immunodepression to better predict mortality.

Given that it remains unclear whether an easy-to-apply nutritional intervention could change the course of HIV infection (26), our objective was first to provide useful tools for physicians working in sub-Saharan Africa to better evaluate the risk of HIV-related mortality. Despite the limitations of the methods, the proposed thresholds have been shown to have a significant prognostic value on survival in our population. If confirmed in other populations, measurement of these anthropometric indicators could become part of the routine clinical evaluation of $\mathrm{HIV}^{+}$patients examined for the first time in sub-Saharan Africa, and the proposed thresholds could contribute to the decision to initiate some interventions, such as chemoprophylaxis of opportunistic infections, based on clinical criteria, whether or not the $\mathrm{CD}^{+}$count is available (18).

\section{ACKNOWLEDGMENTS}

This study was conducted as part of the COTRIMO-CI -ANRS 059 trial and was supported by 1) the Agence Nationale de Recherches sur le SIDA (ANRS, France), the French Ministry of Cooperation, and the Programme National de Lutte contre le SIDA, les MST et la Tuberculose (PNLS/MST/Tub, Côte d'Ivoire) within the "Programme PAC-CI"; and 2) the Agence Francophone pour l'Enseignement Supérieur et la Recherche (AUPELFUREF, France).

The authors thank all the members of the COTRIMO-CI Study Group in Abidjan and Bordeaux. Special thanks are extended to Luc Letenneur (INSERM U.330) for his statistical advice and for reviewing the manuscript.

The COTRIMO-CI Study Group is constituted as follows. Centre de Diagnostic et de Recherches sur le SIDA (CeDReS), CHU de Treichville, Abidjan, Côte d'Ivoire: Xavier Anglaret, Dominique Bonard, Patrice Combe, Nicole Dogbo, Gwenola Gourvellec, Eugène Messou, Hervé Menan, Timothée Ouassa, Fatoumata Sylla-Koko, Siaka Touré, and Lambert Zio-Diakité; Unité INSERM 330, Université Victor Segalen Bordeaux 2, Bordeaux, France: Katia Castetbon, Geneviève Chêne, François Dabis, Sylviane Lafont, Régis Lassalle, Valériane Leroy, and Roger Salamon; Formations Sanitaires Urbaines de Yopougon Attié, Yopougon Toit-Rouge, Abobo Doumé, Abobo Sagbé, et CNPS de Yopougon: Soltié Coulibaly-Koné, Jacob Gnangbomon, David Hamien, Karamoko Koné, and Dorothée Koumi-Meledje; Service de Médecine, $\mathrm{CHU}$ de 
Yopougon, Abidjan, Côte d'Ivoire: Alain Attia, Alassane Mahassadi, Kassi Manlan, and Thérèse N'Dri-Yoman; and Service des Maladies Infectieuses et Tropicales, $\mathrm{CHU}$ de Treichville, Abidjan, Côte D'Ivoire: Eba Aoussi and Makan Coulibaly.

\section{REFERENCES}

1. Revision of the CDC surveillance case definition for acquired immunodeficiency syndrome. Council of State and Territorial Epidemiologists; AIDS Program, Center for Infectious Diseases. MMWR Morb Mortal Wkly Rep 1987;36(suppl 1): $1 \mathrm{~S}-15 \mathrm{~S}$.

2. Acquired immunodeficiency syndrome (AIDS). Interim proposal for a WHO staging system for HIV infection and disease. Wkly Epidemiol Rec 1990;65:221-8.

3. 1993 revised classification system for HIV infection and expanded surveillance case definition for AIDS among adolescents and adults. MMWR Morb Mortal Wkly Rep 1992;41 (RR-17):1-19.

4. WHO case definitions for AIDS surveillance in adults and adolescents. Wkly Epidemiol Rec 1994;69:273-5.

5. Guenter P, Muurahainen N, Simons G, et al. Relationships among nutritional status, disease progression, and survival in HIV infection. J Acquir Immune Defic Syndr 1993;6:1130-8.

6. Clark RA, Blakley SA, Rice J, et al. Predictors of HIV disease progression in women. J Acquir Immune Defic Syndr Hum Retrovirol 1995;9:43-50.

7. Palenicek JP, Graham NMH, He YD, et al. Weight loss prior to clinical AIDS as a predictor of survival. J Acquir Immune Defic Syndr Hum Retrovirol 1995;10:366-73.

8. Wheeler DA, Gibert CL, Launer CA, et al. Weight loss as a predictor of survival and disease progression in HIV infection. J Acquir Immune Defic Syndr Hum Retrovirol 1998;18:80-5.

9. Maas JJ, Dukers N, Krol A, et al. Body mass index course in asymptomatic HIV-infected homosexual men and the predictive value of a decrease of body mass index for progression to AIDS. J Acquir Immune Defic Syndr Hum Retrovirol 1998;19: 254-9.

10. Lindan CP, Allen S, Serufilira A, et al. Predictors of mortality among HIV-infected women in Kigali, Rwanda. Ann Intern Med 1992;116:320-8.

11. Morabia A, Ross A, Curtin F, et al. Relation of BMI to a dualenergy X-ray absorptiometry measure of fatness. Br J Nutr 1999;82:49-55.

12. Sharkey SJ, Sharkey KA, Sutherland LR, et al. Nutritional sta- tus and food intake in human immunodeficiency virus infection. J Acquir Immune Defic Syndr 1992;5:1091-8.

13. Mulligan K, Tai VW, Schambelan M. Cross-sectional and longitudinal evaluation of body composition in men with HIV infection. J Acquir Immune Defic Syndr Hum Retrovirol 1997; 15:43-8.

14. Kotler DP, Tierney AR, Wang J, et al. Magnitude of body-cellmass depletion and the timing of death from wasting in AIDS. Am J Clin Nutr 1989;50:444-7.

15. Paton NIJ, Macallan DC, Jebb SA, et al. Longitudinal changes in body composition measured with a variety of methods in patients with AIDS. J Acquir Immune Defic Syndr Hum Retrovirol 1997;14:119-27.

16. Süttmann U, Ockenga J, Selberg O, et al. Incidence and prognostic value of malnutrition and wasting in human immunodeficiency virus-infected outpatients. J Acquir Immune Defic Syndr Hum Retrovirol 1995;8:239-46.

17. Ott M, Fischer H, Polat H, et al. Bioelectrical impedance analysis as a predictor of survival in patients with human immunodeficiency virus infection. J Acquir Immune Defic Syndr Hum Retrovirol 1995;9:20-5.

18. Anglaret X, Chêne G, Attia A, et al. Early chemoprophylaxis with trimethoprim-sulphamethoxazole for HIV-1-infected adults in Abidjan, Côte d'Ivoire: a randomised trial. Lancet 1999;353:1463-8.

19. Report of a WHO Expert Committee. Physical status: the use and interpretation of anthropometry. Geneva, Switzerland: World Health Organization, 1995.

20. Durnin JVGA, Womersley J. Body fat assessment from total body density and its estimation from skinfold thickness: measurements on 481 men and women aged from 16 to 72 years. Br J Nutr 1974;32:77-97.

21. Everitt B. Cluster analysis. London, England: Halsted Press, 1980.

22. Marubini E, Valsecchi MG. The study of prognostic factors and assessment of treatment effect. In: Marubini E, Valsecchi M, eds. Analysing survival data from clinical trials and observational studies. New York, NY: John Wiley \& Sons, Inc, 1995:295-327.

23. Heymsfield SB, McManus C, Smith J, et al. Anthropometric measurement of muscle mass: revised equations for calculating bone-free arm muscle area. Am J Clin Nutr 1982;36:680-90.

24. Castetbon K, Kadio A, Bondurand A, et al. Nutritional status and dietary intakes in human immunodeficiency virus (HIV)infected outpatients in Abidjan, Côte d'Ivoire, 1995. Eur J Clin Nutr 1997;51:81-6.

25. Grinspoon S, Corcoran C, Miller K, et al. Body composition and endocrine function in women with acquired immunodeficiency syndrome wasting. J Clin Endocrinol Metab 1997;82:1332-7.

26. Macallan DC. Wasting in HIV infection and AIDS. J Nutr 1999;129(suppl):238S-42S. 